\title{
The effect of various doses of a milk protein hydrolysate on the post-prandial glycaemic response in a healthy male cohort
}

\author{
L. Keane and E.R. Gibney \\ UCD Institute of Food and Health, School of Agriculture and Food Science, University College Dublin, Belfield, \\ Dublin 4, Republic of Ireland
}

Type 2 Diabetes is somewhat of a global epidemic, where more than 422 million people worldwide are living with diabetes over the age of $18^{(1)}$. It is evident that a product to target such a non-communicable disease is necessary as a means to improve these statistics. At present, through a previous glucose study carried out by Food for Health Ireland, it is known that $12 \mathrm{~g}$ of a milk hydrolysate effectively reduces the post-prandial glycaemic response, through the action of insulin ${ }^{(2)}$. However, due to the high level of bitterness and the high cost of such hydrolysates, it is important to reduce the required dose while still maintaining efficacy, in order to allow for successful incorporation into food products ${ }^{(3)}$. The aim of the present study was to establish a minimum dose of the milk hydrolysate that would effectively reduce the glycaemic response after a standardised breakfast, compared to $12 \mathrm{~g}$ of the milk hydrolysate in a healthy male cohort.

Participants ( $n$ 13) came to UCD to trial 4 different doses of the milk hydrolysate: $12 \mathrm{~g}, 9 \mathrm{~g}, 6 \mathrm{~g}$ and a control ( $0 \mathrm{~g}$ ). Plasma and serum samples were taken at fifteen-minute intervals for the first hour, and thirty-minute intervals for the second hour of each visit. Serum insulin was analysed using the human insulin ELISA kit (Mercodia, Sweden). Plasma glucose was measured using the Randox clinical chemistry analyser (Randox Laboratories Ltd. United Kingdom). Differences in glucose and insulin concentrations were determined through one-way ANOVA analysis (SPSS 20.0.0).

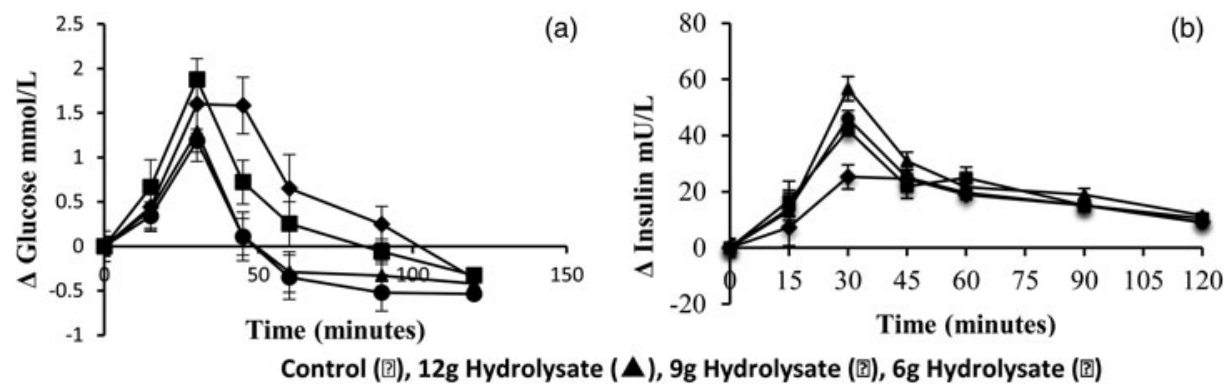

Fig. 1. $\Delta$ Glucose over time (a), $\Delta$ Insulin over time (b)

A significant difference in glucose levels was observed at T45 $(\mathrm{p}<0.005)$. The difference between treatments was observed between the $0 \mathrm{~g}$ and $12 \mathrm{~g}$ of hydrolysate $(\mathrm{p}=0.001)$ and the control and $9 \mathrm{~g}$ of hydrolysate $(\mathrm{p}=0.003)$. No significant difference was seen using repeated measures analysis. One-way ANOVA analysing $\Delta$ glucose showed that a significant difference was seen between T0 - T45 and T60 respectively ( $\mathrm{p}=0.001$ and $\mathrm{p}<0.05$ respectively) (Fig. 1a). At T0-T60, the significant difference was observed between 0 g and both the $12 \mathrm{~g}$ and $9 \mathrm{~g}$ of the milk hydrolysate. A significant difference was also observed using repeated measures analysis ( $\mathrm{p}<$ 0.005). With regards to insulin concentration, a limited significant difference was observed between $12 \mathrm{~g}$ and $0 \mathrm{~g}$ at $\mathrm{T} 15 \mathrm{and} \mathrm{T} 30$ (Fig. 1b).

Results demonstrate that $12 \mathrm{~g}$ and $9 \mathrm{~g}$ of the milk hydrolysate are effective at lowering blood sugars. However, the $12 \mathrm{~g}$ dose of the milk hydrolysate was marginally more potent in comparison to the $9 \mathrm{~g}$ dose. No overall significant difference was observed in insulin concentration. These results warrant further investigations of the $12 \mathrm{~g}$ and $9 \mathrm{~g}$ doses in additional cohorts such as those with abnormal glucose metabolism.

1. WHO. Global Report on Diabetes. France: World Health Organisation; 2016.

2. Pasin G, Comerford KB. (2015) Dairy foods and dairy proteins in the management of type 2 diabetes: a systematic review of the clinical evidence. Adv Nutr 6, 245-259.

3. Paul M, Somkuti GA. (2009) Degradation of milk-based bioactive peptides by yogurt fermentation bacteria. Lett Appl Microbiol 49, 345-350. 